\title{
Anomalous Origin of the Coronary Arteries: An Account of Six Cases
}

\author{
Laxman Dubey' \\ 'Department of Cardiology, College of Medical Sciences and Teaching Hospital, Bharatpur, Chitwan, Nepal.
}

\section{ABSTRACT}

Anomalous origin of coronary arteries is a rare type of congenital anomalies with a low incidence. It is considered an incidental finding without clinical significance, however, these abnormalities may be responsible for angina pectoris, heart failure, arrhythmias and increased risk of sudden death. In this paper, six cases that were diagnosed with anomalous origin of coronary arteries confirmed by coronary angiography has been described and also briefly reviewed the literature concerning current views and therapy of this abnormality.

Keywords: anomalous origin of coronary artery; coronary angiography; incidence.

\section{INTRODUCTION}

Congenital anomalies of the coronary arteries are present in about $1.3 \%$ of adults undergoing cardiac catheterization. ${ }^{1}$ Anomalous origin of the coronary arteries (ACAO) could be considered an incidental finding without clinical significance, ${ }^{2}$ however, depending upon the origin, course, and termination of the anomalous vessel, these abnormalities may be responsible for angina pectoris, heart failure and sudden death. Therefore, accurate recognition and documentation of ACAO at the time of coronary angiography (CAG) are essential to determine the significance of such findings and to avoid therapeutic complications. In this case study, six cases of ACAO identified during retrospective analysis of all coronary angiography (CAG) $(n=450)$ performed during 2011 to 2013 are reported.

\section{CASE 1}

A 43 years old man was admitted to our hospital complaining of acute chest pain for six hours duration. His medical history was not significant. He was a chronic smoker. On admission, his electrocardiogram (ECG) showed ST-segment elevation in leads II, III and aVF. CK-MB was 58U/L and troponin I was positive. CAG was performed via right femoral artery approach.
Cannulation of the left coronary artery (LCA) displayed normal courses of the left main coronary artery (LM), normal left circumflex artery (LCX) and a significant obstructive lesion (90\%) in the proximal part of the left anterior descending artery (LAD) just before the first diagonal branch, with good distal flow. Attempts to cannulate the right coronary artery (RCA) with the right Judkins catheter were unsuccessful. Selective angiography of the RCA was successful with 6 French Xtra Backup XB 3.5 guiding catheter with slight clockwise rotation, which revealed anomalous high anterior takeoff of the RCA with total occlusion at the mid level (Figure 1a, 1b).

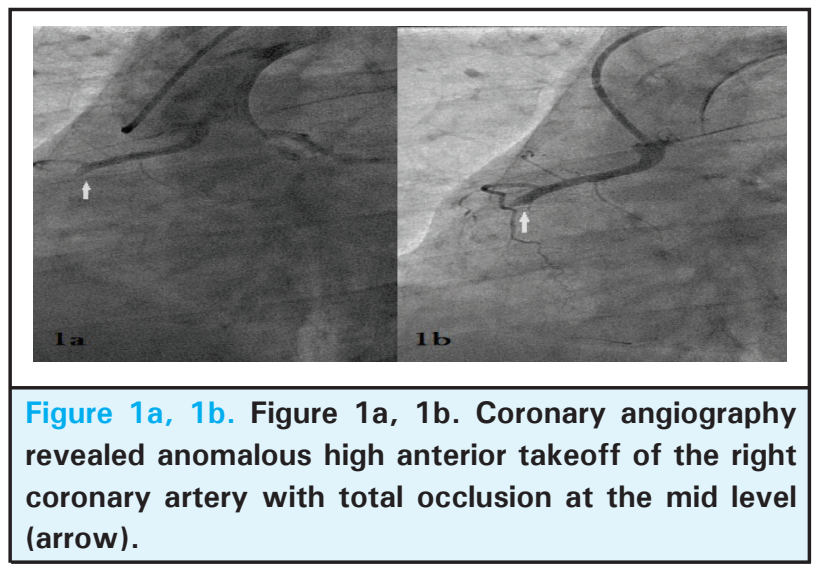

Correspondence: Dr. Laxman Dubey, Department of Cardiology, College of Medical Sciences and Teaching Hospital, Bharatpur, Chitwan, Nepal. Email: dubeylax@yahoo.com, Phone: 9851123288. 


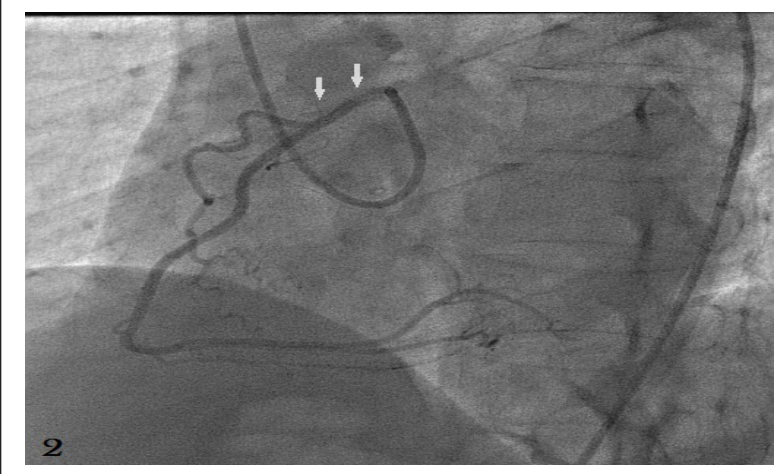

Figure 2. Selective cannulation of the right coronary artery revealed right coronary artery originating from the left sinus (arrow).

Primary percutaneous coronary interevention (PCI) and stenting to the RCA was done with final good end result achieving Thrombolysis in Myocardial Infarction (TIMI) III flow. The patient's subsequent hospital stay was uneventful and discharged home free of angina four days later. Two weeks later, elective $\mathrm{PCl}$ to the LAD was performed.

\section{CASE 2}

A 52 years old man with no relevant medical history presented with left side chest heaviness and shortness of breath on exertion. The patient was an active individual and had not previously had such problems on exertion. The presenting symptoOms had begun approximately seven days before. The episode lasted a few minutes but resolved spontaneously. An ECG showed normal sinus rhythm without any evidence of ischemic changes. Cardiac enzymes were negative. CAG was performed via right femoral artery approach. Selective cannulation of the left coronary system showed normal LCA. However, selective cannulation was difficult for the RCA. Non selective angiography revealed RCA originating from the left sinus. Selective cannulation of the RCA was possible by Extra BackUp guiding catheter which revealed RCA originating from the left sinus but without any obstructive lesion (Figure 2).

\section{CASE 3}

A 39 years old male presented to our center with acute central chest pain of five hours duration. The patient was an active individual and had exertional chest heaviness since last four days. He was a smoker with negative history of hypertension and diabetes mellitus. On arrival his BP was $110 / 70 \mathrm{mmHg}$, pulse rate of 90 beats per minutes. ECG showed ST-segment elevation in leads V1-V4 with ST segment depression in the inferior leads. $\mathrm{He}$ was diagnosed to have acute STEMI and taken to the catheterization laboratory. CAG was performed via right femoral approach. Selective cannulation of the $\mathrm{LCA}$ revealed $95 \%$ stenosis in the mid LAD with good distal flow, however, RCA cannulation was not successful. Non-selective angiography revealed the RCA originating from the left coronary sinus (Figure 3). Patient underwent primary $\mathrm{PCl}$ stenting to the LAD (culprit lesion). His subsequent hospital stay was uneventful and discharged after five days.

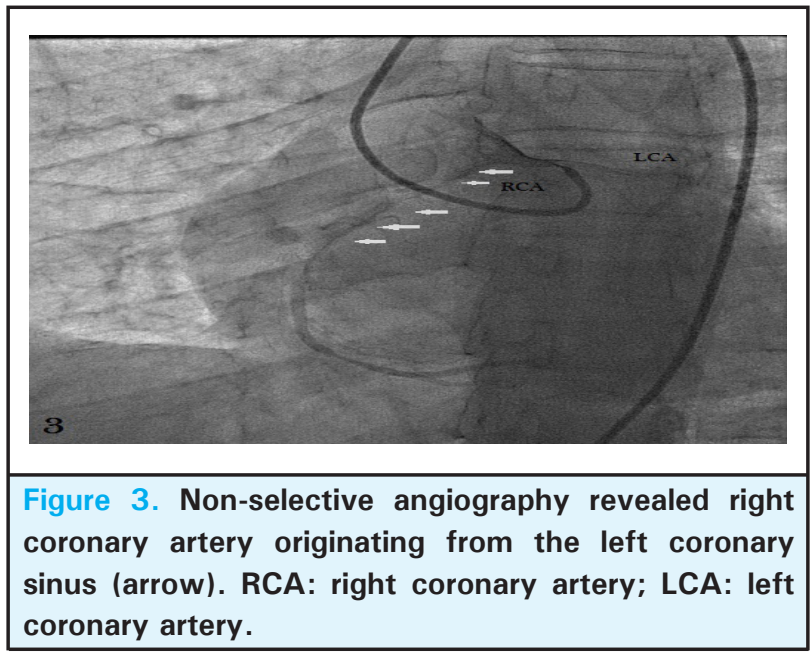

\section{CASE 4}

A 47 years old male presented to our emergency room with acute chest pain associated with profuse sweating and one episode of vomiting. The pain lasted for three hours which was left sided. His medical history was not significant. He was a smoker. His BP was 120/60 $\mathrm{mmHg}$, heart rate of 110 beats per minute. ECG showed sinus rhythm with ST-segment elevation in leads V2-V6, lead I and aVL. CK-MB was $108 \mathrm{U} / \mathrm{L}$ and troponin I was positive. He was diagnosed to have an acute extensive anterior wall $\mathrm{Ml}$ and transferred to the catheterization laboratory for primary $\mathrm{PCl}$. Selective cannulation of the LCA by Judkins left catheter revealed total obstruction at the proximal LAD. In the same injection, it was noted that a dominant anomalous RCA was also arising from the left aortic sinus (Figure 4). Primary $\mathrm{PCl}$ stenting to the LAD was done. His stay in the hospital was uneventful and discharged home free of angina four days later. 


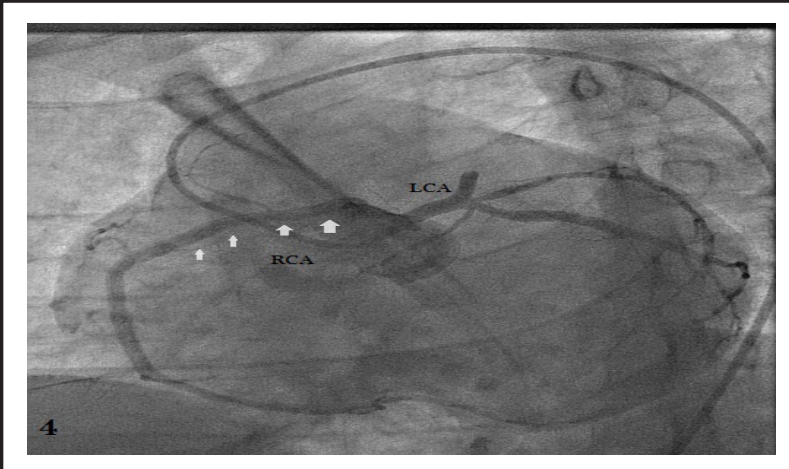

Figure 4. Coronary angiography revealed total occlusion of the left anterior descending artery and dominant right coronary artery (arrow) was also arising from the left aortic sinus. RCA: right coronary artery; LCA: left coronary artery.

\section{CASE 5}

A 41 years old male patient was referred to our center for evaluation of chest pain. He had typical exertional chest pain which started since last one month. An ECG showed normal sinus rhythm without any evidence of ischemic changes, however, repeat ECG during chest pain showed ST segment depression with $\mathrm{T}$ inversion in leads V2-V5. The blood cardiac enzymes were negative. His medical history showed that he was a non-smoker with no significant indicators for diabetes mellitus, hypertension, or hyperlipidemia. On admission, a physical examination revealed that his vital signs were within normal limits. CAG was performed via right femoral approach. Selective cannulation of the LCA revealed single LM coronary artery giving the LAD branch only, the LCX was absent. The LAD was diffusely diseased with subtotal obstruction starting from the mid to the distal segment (Figure 5a).

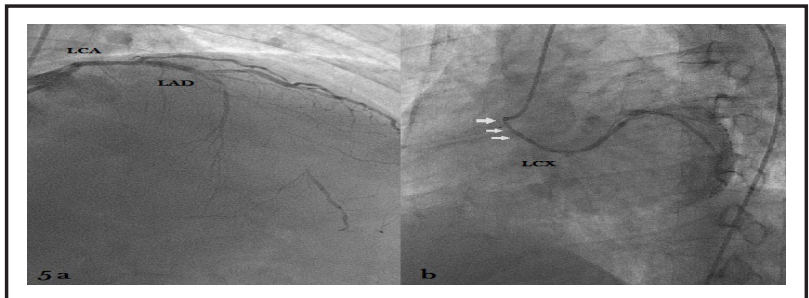

Figure 5a. Left main coronary artery giving the left anterior descending artery only, Figure $5 \mathrm{~b}$. Anomalous origin of the left circumflex artery from the right sinus (arrow). LCA: left coronary artery; LAD: left anterior descending artery; LCX: left circumflex artery.

RCA was normal. Selective cannulation of the LCX was possible by right Judkins catheter which revealed anomalous origin of the LCX from the right sinus with no significant obstructive lesion (Figure $5 b$ ). LAD lesion was not suitable for $\mathrm{PCl}$, and the patient was referred for possible coronary artery bypass graft (CABG) surgery. CABG was performed one month later; left internal mammary artery to the LAD and saphenous venous to the diagonal branch grafted and the patient remained asymptomatic during six month follow-up.

\section{CASE 6}

A 48 years old female presented to our center with exertional chest pain one week duration which had increased in intensity for one day. Pain was associated with sweating. She had a history of systemic hypertension but was not on medication. Her ECG showed sinus rhythm with non-specific T-wave abnormalities. On clinical examination, her BP was $140 / 80 \mathrm{mmHg}$ and a regular pulse of 78 beats per minute. Hemoglobin, white cell count, differential white cell count, serum electrolytes, renal function tests, and liver function tests were within normal limits. CK-MB was $28 \mathrm{U} / \mathrm{L}$ and troponin I was negative. Cardiac catheterization was done via right radial artery approach. Selective catheterization of the LCA showed normal LM, normal LCX, and a significant obstructive lesion $(80 \%)$ in mid part of the LAD just after the first diagonal branch, with good distal flow. During selective catheterization of the LCA, filling in the RCA was noted. Attempted cannulation of the RCA was difficult. Nonselective angiography revealed aberrant dominant RCA arising from the left aortic sinus adjacent to the origin of the LCA (Figure 6a).

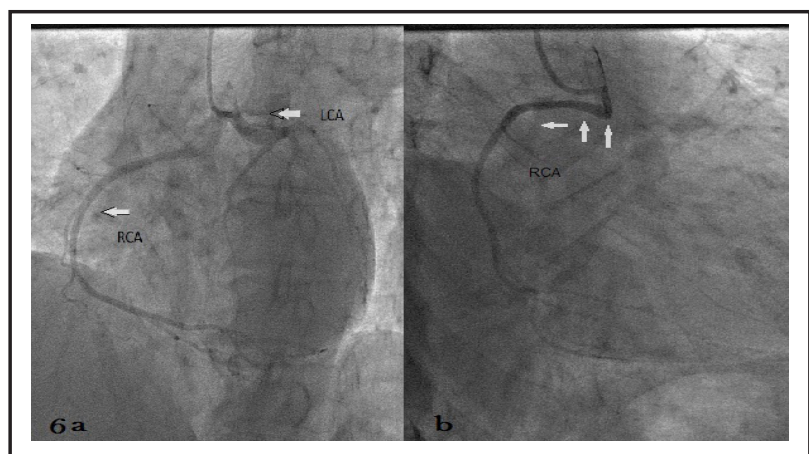

Figure 6a. Nonselective angiography revealed aberrant dominant right coronary artery arising from the left aortic sinus adjacent to the origin of the left coronary artery, Figure $6 \mathrm{~b}$. Selective angiography of the right coronary artery showed anomalous origin from left aortic sinus. RCA: right coronary artery; LCA: left coronary artery.

Selective angiography of the RCA showed anomalous origin from left aortic sinus; however, the RCA had no significant stenotic lesions (Figure 6b). The diagnosis of obstructive LAD lesion with anomalous origin of the RCA from the left aortic sinus was made. She underwent $\mathrm{PCl}$ 
and stenting to the LAD. Her subsequent hospital stay was uneventful, and she was discharged home free of angina three days later. At three month follow-up, patient remained asymptomatic.

\section{DISCUSSION}

Congenital anomalies of the coronary arteries are present in about $1.3 \%$ of adults undergoing cardiac catheterization. ${ }^{1}$ There are two types of ACAO: from the pulmonary artery, and from the aorta. The most common anomaly is aberrant origin of the LM or RCA from the wrong sinus of Valsalva. An anomalous origin of the LAD is one of the rarest anomalies. The LAD can arise from the right coronary sinus or RCA and take a course anterior to the right ventricular outflow tract or it may take an anomalous proximal course. The LCX can arise from the right sinus of Valsalva or the RCA. In the Coronary Artery Surgery Study, ${ }^{3}$ which included CAG of 24,959 patients, the most common anomaly involved the LCX $(60 \%)$. In $69 \%$ of these cases, the LCX arose from a separate ostium in the right coronary sinus of Valsalva, and in $31 \%$ of cases it originated as a branch of the RCA. However, in other reports anomalous RCA was considered the most common one. The incidence of anomalous RCA originating from the left coronary system ranges from $0.1 \%$ to $0.9 \%$. The origin of an anomalous RCA may be from the left sinus of Valsalva, the posterior sinus of Valsalva, the ascending aorta, the PA, the left ventricle, the LM, the LCX or the LAD. Origin of the LCA from the pulmonary trunk is a rare finding in adults who are undergoing CAG for suspected ischemic heart disease. In rare instances, all three coronary arteries arise from the single coronary sinus. Only a small number of case reports, ${ }^{4}$ have described this anomaly.

Among 450 CAG performed till 2013, six patients had ACAO with angiographic incidence of $1.4 \%$. All of them were adults with ages ranging from 39 to 52 years (mean age, 45 years). Anomalous origin of the RCA was the most common coronary anomaly, in particular anomalous origin of the RCA from opposite sinus was the most common (66.7\%) with angiographic incidence of $0.9 \%$, high anterior takeoff origin of the RCA was present in $16.7 \%$ (angiographic incidence of $0.3 \%$ ) and the LCX arising from the right sinus in $16.7 \%$ (angiographic incidence of $0.3 \%$ ) (Table 1 ).

\begin{tabular}{|llll|}
\hline $\begin{array}{l}\text { Table 1. Incidence of different congenital coronary } \\
\text { artery anomalies in angiographic population (total of } \\
450 \text { patients). }\end{array}$ \\
\hline $\begin{array}{l}\text { Coronary } \\
\text { anomaly }\end{array}$ & $\begin{array}{l}\text { Number } \\
\text { of } \\
\text { patient }\end{array}$ & $\begin{array}{l}\text { Angiographic } \\
\text { incidence } \\
(\%)\end{array}$ & $\begin{array}{l}\text { Anomaly } \\
\text { incidence } \\
\text { (\%) }\end{array}$ \\
$\begin{array}{l}\text { RCA arising } \\
\text { from left } \\
\text { sinus }\end{array}$ & 4 & $0.9 \%$ & $66.7 \%$ \\
$\begin{array}{l}\text { High anterior } \\
\text { takeoff origin } \\
\text { of RCA }\end{array}$ & 1 & $0.3 \%$ & $16.7 \%$ \\
$\begin{array}{l}\text { LCX arising } \\
\text { from right } \\
\text { sinus }\end{array}$ & 1 & $0.3 \%$ & $16.7 \%$ \\
$\begin{array}{l}\text { All } \\
\text { anomalous } \\
\text { coronary } \\
\text { artery }\end{array}$ & 6 & $1.4 \%$ & $100 \%$ \\
\hline
\end{tabular}

Patients with ACAO may remain asymptomatic or they die suddenly especially at a young age and after extreme exertion. ${ }^{5}$ Most patients are asymptomatic for a large portion of their lives, and incidentally identified during CAG for an atypical chest-pain. Whether the presence of an ACAO increases the chances of CAD remains controversial. Some have shown significantly smaller incidence of coronary stenosis in anomalous versus normal vessels and the anomalous arteries are relatively protected from stenotic disease. ${ }^{6,7}$ However, anomalous vessels seem to develop earlier and greater atherosclerotic lesions than normal ones. Patients with the ACAO are more likely to have symptoms of myocardial ischemia and researchers have found associations between this anomaly and acute $\mathrm{MI}$, angina pectoris, syncope, malignant arrhythmias, and sudden death, in the absence of atherosclerotic or other cardiac disease. The ischemia in patient with non-atherosclerotic ACAO could be secondary to a combination of several factors that produce obstruction, such as mechanical compression of the anomalous artery by the great vessels or the oblique angle at the juncture of the anomalous coronary artery and a slitlike orifice in the aortic wall, which can collapse during exertion. ${ }^{8}$

In our series, all patients presented with symptoms of myocardial ischemia. Among them, four patients who presented with acute $\mathrm{Ml}$ and rest two presented with exertional chest discomfort associated with shortness of breath. Interestingly, one patient had no atherosclerotic disease, four had significant obstructive disease in non 
anomalous coronary artery and only one patient had total occlusion of the anomalous coronary artery.

Patients with ACAO have three treatment options: medical treatment, $\mathrm{PCl}$ and stenting, and surgical repair. ${ }^{5}$ However, the long-term benefits of such therapies have not yet been demonstrated. Surgery in patients older than 35 years is not advised by some authors because sudden death mostly occurs before 35 years of age and the risk of sudden death decreases with age..$^{9,10}$ In such cases the chief aim is good symptomatic relief. In a five year follow-up study in congenital coronary anomaly managed medically, no death was found to be directly related to the congenital anomaly. ${ }^{8}$ When revascularization is needed, $\mathrm{PCl}$ would be the first choice similar in non-anomalous origin coronary artery. However, doing $\mathrm{PCl}$ in $\mathrm{ACAO}$ is technically challenging such as frequent catheter exchanges before finding the best-fitting catheter and guidewire because of difficulty in selective cannulation and optimal support, giving rise to increased fluoroscopic time and a greater amount of contrast agent used during $\mathrm{CAG}$ and $\mathrm{PCI}$. In patient who cannot be effectively treated by $\mathrm{PCl}, \mathrm{CABG}$ surgery is of vital importance in the therapy of a coronary anomaly. In conclusion, ACAO is a rare type of congenital anomalies. These abnormalities may be responsible for angina pectoris, heart failure and sudden death. Therefore, accurate recognition and documentation of coronary artery anomalies at the time of CAG are essential to determine the significance of such findings and to avoid therapeutic complications.

\section{REFERENCES}

1. Yamanaka O, Hobbs RE. Coronary artery anomalies in 126,595 patients undergoing coronary arteriography. Cathet Cardiovasc Diagn. 1990;21:28-40.

2. Dubey L. Percutaneous coronary intervention of an obstructive left anterior descending artery with anomalous origin of right coronary artery. ARYA Atheroscler. 2013;9:164-6.

3. Click RL, Holmes DR Jr, Vlietstra RE, KosinskiAS, Kronmal RA. Anomalous coronary arteries: location, degree of atherosclerosis and effect on survival- a report from the Coronary Artery Surgery Study. J Am Coll Cardiol. 1989;13:531-7.

4. Neil DA, Bonser RS, Townend JN. Coronary arteries from a single coronary ostium in the right coronary sinus: a previously unreported anatomy. Heart. 2000;83:E9.

5. Angelini P. Coronary artery anomalies: an entity in search of an identity. Circulation. 2007;115:1296-305.
6. Topaz O, DeMarchena EJ, Perin E, Sommer LS, Mallon SM, Chahine RA. Anomalous coronary arteries: angiographic findings in 80 patients. Int J Cardiol. 1992;34:129-38.

7. Zhang F, Ge JB, Qian JY, Fan B, Wang QB, Chen HZ. Frequency of the anomalous coronary origin in the Chinese population with coronary artery stenosis. Zhonghua Nei Ke Za Zhi. 2005;44:347-9.

8. Kaku B, Shimizu M, Yoshio H, Ino H, Mizuno S, Kanaya $\mathrm{H}$, et al. Clinical features of prognosis of Japanese patients with anomalous origin of the coronary artery. Jpn Circ J. 1996;60:731-41.

9. Shah AS, Milano CA, Lucke JP. Anomalous origin of the right coronary artery from the left coronary sinus: case report and review of surgical treatments. Cardiovasc Surg. 2000;8:284-6.

10. Hamzeh G, Crespo A, Estaran R, Rodriguez MA, Voces R, Aramendi JI. Anomalous origin of right coronary artery from left coronary sinus. Asian Cardiovasc Thorac Ann. 2008;16:305-8. 